\title{
Polifonía discursiva y procesos de cambio en los formadores de docentes: ¿Qué voces movilizan sus prácticas de enseñanza?*
}

\author{
Discursive polyphony and changes in future teachers' educators: which voices \\ move their teaching practices?
}

\section{Poifonia discursiva e processos de transformações nos formadores de professores: Que vozes mobilizam suas práticas de ensino?}

\author{
Helena Montenegro Maggio, ${ }^{a}$ Lorena Medina Morales ${ }^{b}$
}

aPontificia Universidad Católica de Chile. Correo electrónico: hlmonten@uc.cl bPontificia Universidad Católica de Chile, Facultad de Educación. Telf.: 5623545387.

Correo electrónico: lmedinam@uc.cl

\begin{abstract}
RESUMEN
Este artículo reporta los principales resultados de un estudio orientado a develar el discurso pedagógico que subyace a la modalidad de enseñanza que implementan los formadores de futuros profesores. Para ello se propone un modelo conceptual que permita comprender las prácticas de enseñanza que se implementan en los procesos formativos y que se configuran por medio de un discurso pedagógico. La recolección de los datos se obtuvo por medio de entrevistas en profundidad efectuadas a siete docentes formadores pertenecientes a cuatro carreras de Pedagogía en Enseñanza Básica. Las entrevistas fueron analizadas por medio del análisis dialógico del discurso (ADD). Los resultados obtenidos indican la coexistencia de distintas prácticas de enseñanza entre los formadores de acuerdo al modelo que se propone, y que oscilan de menor a mayor grado de complejidad y alteridad. Finalmente, el artículo concluye con las implicancias prácticas e investigativas de estos resultados en la formación docente inicial.
\end{abstract}

Palabras Clave: formador, prácticas de enseñanza, discurso pedagógico, análisis dialógico del discurso, dialogicidad.

ABSTRACT
This article reports the main findings of a study aiming to explore the pedagogical discourse hold by educators teaching future teachers. Consequently, the study proposes a conceptual model with the main purpose of understanding the approaches to teaching in teachers' education through pedagogical discourse. Data was collected through semi-structured, in-depth interviews conducted face-to-face with seven teacher educators from four pre-service Teacher Education Programs. The interviews were analyzed using the Dialogic Discourse Analysis Method (DDA). Results showed that approaches to teaching coexist among teacher educators according to the conceptual model proposed, ranging from a low to a high degree of complexity and alterity (otherness). Finally, the article concludes that these findings have implications for both practice and research in teacher education.

Key Words: teacher educators, approaches to teaching, pedagogical discourse, dialogic discourse analysis, dialogism.

\section{RESUMO}

Apresentam-se os principais resultados de um estudo voltado para desvelar o discurso pedagógico que subjaz à modalidade de ensino implementada por formadores de futuros professores. Para isso, um modelo conceitual é proposto a fim de entender as práticas de ensino que são implementadas nos processos educativos e que se

Estudio financiado por CONICYT, mediante la Beca de Apoyo a la Realización de Tesis Doctoral AT 24100198: "La práctica de enseñar en los Programas de Formación Docente Inicial: ¿Cómo el profesor se convierte en formador de futuros profesores?”. 
Estudios Pedagógicos, vol. XL, Número Especial 1: 161-182, 2014

POLIFONÍA DISCURSIVA Y PROCESOS DE CAMBIO EN LOS FORMADORES DE DOCENTES: ¿QUÉ VOCES

MOVILIZAN SUS PRÁCTICAS DE ENSEÑANZA?

configuram por meio de um discurso pedagógico. A coleta de dados foi obtida por meio de entrevistas em profundidade de sete formadores de professores de quatro carreiras da Pedagogia do Ensino Fundamental. As entrevistas foram analisadas por meio da análise dialógica do discurso (ADD). Resultados obtidos mostram que, de acordo com o modelo proposto, coexistem diferentes práticas de ensino entre os formadores e que oscilam de menor a maior grau de complexidade e alteridade. Por fim, conclui-se com as implicações práticas e de pesquisa desses resultados na formação inicial de professores.

Palavras-chave: formadores, práticas de ensino, discurso pedagógico, análise dialógica do discurso, dialogicidade.

\section{INTRODUCCIÓN}

Un tema clave en las políticas educativas de varios países, entre ellos Chile, ha sido fortalecer la formación docente inicial que se imparte a los futuros profesores (Ávalos, 2001; Darling-Hammond, 2006; Korthagen, Loughran y Russell, 2006; Ministerio de Educación [Mineduc], 2005). La finalidad es formar docentes capaces de enfrentar las demandas del siglo XXI y potenciar aprendizajes significativos en sus estudiantes (Darling-Hammond y Bransford, 2005). De manera particular, uno de los actores que se ha comenzado a investigar con mayor detención son los profesionales que forman a los futuros profesores. Prueba de ello son los distintos estudios y literatura especializada acerca de los educadores responsables de enseñar en los programas de formación docente inicial (Berry, 2007; Cochran-Smith, 2003; Korthagen, Loughran y Lunenberg, 2005; Loughran, 2006; Loughran y Berry, 2005; Murray y Male, 2005; Russell y Loughran, 2007; Swennen y Van der Klink, 2009; Zeichner, 2005). Los resultados de estos estudios han contribuido con cuerpos de conocimientos e implicancias prácticas tanto para los programas de formación como para el aprendizaje profesional de los formadores (Cochran-Smith, 2003; Loughran, 2006; Martinez, 2008).

No obstante ello, en Chile la figura del docente ha sido un tema escasamente abordado, investigado y discutido en el campo de la formación docente inicial (Cisternas, 2011; Cornejo, 2005, 2007; Mineduc, 2005; Montenegro y Fuentealba, 2012; Organización para la Cooperación y el Desarrollo Económicos [OECD], 2004). Por ejemplo, Cisternas (2011) plantea que se estudia un 50\% menos al docente formador que al futuro profesor. Asimismo, estos estudios no se han focalizado en las prácticas pedagógicas que éste adopta y el impacto que generan dichas prácticas en los profesores en formación. Esto cobra relevancia si se toma en cuenta que los formadores poseen una particularidad distintiva: cuando enseñan la profesión docente al mismo tiempo la modelan a través de su propia práctica. Es decir, enseñan y enseñan a enseñar en forma simultánea a los futuros profesores (Loughran, 2006; Loughran y Berry, 2005; Lunenberg, Korthagen y Swennen, 2007). Dada esta complejidad, aproximarse a la problemática de quienes enseñan a enseñar no es una tarea fácil. La gran diversidad de enfoques teóricos coexistentes, la pluralidad de aproximaciones metodológicas y la fragmentación de un campo de investigación emergente son motivos que dificultan su clarificación y sistematización conceptual (Cisternas, 2011; Medina, 2006). Incluso, para una adecuada aproximación a este objeto de estudio, se considera fundamental tener en cuenta tres temas claves en el campo de la investigación de los docentes formadores.

En primer lugar, si bien la enseñanza en el sistema escolar posee elementos comunes con la enseñanza que se imparte en la formación docente inicial, al mismo tiempo cada una de ellas conllevan énfasis distintivos (Dinkelman, Margolis y Sikkenga, 2006). Una 
diferencia importante de señalar se centra en el papel de los educadores. Loughran (2006) destaca que los docente de aula sólo enseñan ("just teaching"), en contraposición a los docentes formadores que "hacen" la enseñanza ("doing teaching"), lo cual implica un mayor grado de complejidad. Esto se puede comprender mejor si se toma en cuenta que el tópico que se enseña e investiga en los programas de formación (en este caso "enseñar") es el mismo que los formadores de futuros profesores realizan cuando imparten clases (Kane, 2007), y que el conocimiento disciplinar y el conocimiento pedagógico se enseñan de manera conjunta cuando los formadores enseñan a enseñar (Loughran, 2006; Murray y Male, 2005). Sin embargo, esta complejidad no se logra articular ni visualizar de manera clara al interior de los programas de formación.

En segundo lugar, Borko (2004) refiere que el aprendizaje profesional se desarrolla de manera situada. En otras palabras, esta autora postula que el desarrollo profesional es un aprendizaje que se da en actividades socialmente organizadas. Para el caso de los formadores, el desarrollo profesional respecto de la enseñanza y el aprendizaje de la enseñanza representa una práctica social que es situada y compartida a través de un discurso pedagógico (Robinson y McMillan, 2006). Vale decir, los significados se construyen y elaboran por medio de un lenguaje particular que se da entre docentes y estudiantes (Montenegro, Medina y Valdivia, 2013).

Finalmente, en tercer lugar, Bajtín (1982) plantea que el lenguaje es intersubjetivo y en referencia a un destinatario. Para este autor el sentido se produce y circula a través del lenguaje que es polifónico por definición ya que materializa un conjunto de voces que provienen de distintos orígenes, tiempos, culturas y contextos sociales inscritos al interior de éste. En cambio, la tendencia en el campo investigativo de los docentes se ha focalizado en el relato de sus experiencias desde una perspectiva de naturaleza más bien monológica, representada principalmente en una voz: el relato de su historia como profesor (Savvidou, 2010). Por ende, falta el desarrollo de estudios de narrativas de profesores desde una perspectiva dialógica, que incorporen a través del análisis de sus discursos, no sólo la voz del docente formador entendida como el resultado de un proceso de formación y desarrollo profesional, sino como la construcción y re-significación constante del tejido discursivo que incorpora, a través diversos mecanismos de relación, las voces internamente convincentes de personas e instituciones que han incidido e inciden en este proceso.

Comprender dialógicamente la formación de profesores a través de los discursos que configuran su historia y procesos es acercarse a la naturaleza de las relaciones humanas en diversas esferas de interacción; en este caso particular, la academia, se deben considerar las características que son inherentes a procesos vistos desde una perspectiva sociocultural: sus tensiones, dinamismos, y configuraciones sociales internalizadas desde la cadena discursiva de voces propias y ajenas, durante la historia de vida en el ámbito estudiado. El supuesto que subyace a esta afirmación es que no sólo se dialoga con otras voces, en una especie de vías paralelas donde a veces se encuentran los discursos unipersonales y monologales, sino que los discursos y las voces o posiciones ideológicas a las que se adhiere, configuran todos y cada uno de los discursos, de modo que es posible adherir o confrontarse atendiendo a la dialogización interna de la palabra o del discurso. Cada discurso pone en juego una verdadera red o cadena de enunciados y permite reconocer las voces ajenas, internamente convincentes, en el propio discurso. En el caso de este trabajo, permite reconocer en los discursos de los formadores aquellas voces que configuran su historia profesional y personal, y el modo en que han entrado en relación para construir su trayectoria profesional. 
Estudios Pedagógicos, vol. XL, Número Especial 1: 161-182, 2014

POLIFONÍA DISCURSIVA Y PROCESOS DE CAMBIO EN LOS FORMADORES DE DOCENTES: ¿QUÉ VOCES MOVILIZAN SUS PRÁCTICAS DE ENSEÑANZA?

Teniendo en cuenta la escasez de estudios acerca de la práctica de enseñar de los formadores desde una perspectiva dialógica, este artículo comparte los resultados de un estudio orientado a explorar el discurso pedagógico que poseen los formadores de futuros profesores. Específicamente, devela los sentidos y significados que siete docentes formadores elaboran respecto de su práctica de enseñar a enseñar. Para el logro de este propósito, este estudio propone un modelo conceptual para comprender las prácticas de enseñanza en los formadores. Asimismo, conceptualiza la enseñanza como un proceso social y culturalmente situado en donde la alteridad (entendida dialógicamente) se configura sistemáticamente al interior del discurso pedagógico.

\section{MARCO DE REFERENCIA}

\subsection{MODELO CONCEPTUAL PARA EL ESTUDIO DE LAS PRÁCTICAS DE ENSEÑANZA EN LOS FORMADORES}

La bibliografía consultada da cuenta de distintos modelos conceptuales vinculados a la modalidad de enseñanza que adoptan docentes y formadores (Cochran-Smith y Lytle, 1999; Hutchings y Shulman, 1999; Shulman, 1987). Sin embargo, en dicha discusión no se aprecian algunos temas significativos de tener en cuenta. Por ejemplo, si bien estas propuestas indican diversas conceptualizaciones acerca del conocimiento a la base de la enseñanza, no explicitan en forma clara cómo dichos conocimientos se manifiestan cuando los profesores y docentes formadores se enfrentan a la experiencia de enseñar. A su vez, tampoco se aprecia de qué forma el tipo de conocimiento a la base de la enseñanza se transfiere desde el docente formador al futuro profesor en formación.

Como una forma de contribuir en esta discusión, este estudio propone un modelo conceptual para investigar las prácticas de enseñanzas que adoptan los formadores de futuros profesores desde tres enfoques: con énfasis (1) en la Transmisión de Conocimientos; (2) en el desarrollo de un Conocimiento Pedagógico del Contenido; y (3) en el Desarrollo de un Conocimiento Académico de la Enseñanza (Montenegro y Fuentealba, 2012). Estos tres tipos de prácticas de enseñanza coexisten al interior de los programas de formación y representan distintos posicionamientos y formas de llevar a cabo la enseñanza. De igual modo, cada una de ellas presupone diferentes formas de comprender el proceso de enseñanza que va de menor a mayor complejidad. A su vez, teniendo en cuenta que los docentes formadores además de enseñar contenidos enseñan a enseñar (Loughran, 2006), este modelo apuesta a que la forma de abordar la enseñanza que adopta el formador se manifiesta en estos dos procesos formativos. Es decir, si en un formador prevalece una práctica de enseñanza, esta se manifiesta en correspondencia con dicho enfoque cuando enseña y enseña a enseñar (Dall' Alba y Sandberg, 2006).

Una práctica de enseñanza con énfasis en la transmisión de conocimientos (PETC) considera la enseñanza como una actividad genérica que se da con independencia del contexto en que se desarrolla (Shulman, 2004). El foco está en la transmisión de información hacia los estudiantes sustentada desde una racionalidad técnica (Brew, 2006; Bullock, 2009; Loughran y Russell, 2009). Por ende, se concibe la formación de los profesores como la acumulación de una serie de contenidos y procedimientos para ser reproducidos con posterioridad cuando se desempeñe profesionalmente (Loughran, 
2006). La bibliografía consultada da cuenta que este tipo de práctica de enseñanza es bastante común al interior de los programas de formación de profesores. Por ejemplo, cuando se consignan modelos de enseñanza centrados en la transmisión de contenidos e información (Berry, 2007, 2009; Loughran y Russell, 2009; OECD, 2004). Con relación a las prácticas pedagógicas que se adoptan, se caracteriza por un formador que "habla" a un futuro profesor que "escucha" (Berry, 2009; Bullock, 2009). En otras palabras, cuando el foco está en los contenidos transmitidos por la persona que enseña, más que vincular esa información con los conocimientos previos que posee el estudiante (Loughran \& Russell, 2009), se parte de la base de que si dicha transmisión se realiza de manera adecuada, el aprendizaje se desarrollará de manera fluida y natural en el estudiante (Loughran, 2006).

En cambio, una práctica de enseñanza con énfasis en el desarrollo de un conocimiento pedagógico del contenido (PE-CPC) permite distinguir entre un formador y un especialista en la materia (Shulman, 1987). El conocimiento pedagógico del contenido se define como la capacidad para transformar el conocimiento de un contenido en una modalidad pedagógica pertinente a las habilidades y capacidades de los estudiantes (Depaepe, Verschaffel y Kelchtermans, 2013; Shulman, 1986, 1987). Al interior de los programas de formación se ha investigado esta modalidad de enseñanza a través del estudio de las estrategias de enseñanza que adopta el formador y su relación con el aprendizaje que desarrolla el estudiante en formación (Lunenberg y Korthagen, 2003; Struyven, Dochy y Janssens, 2010). Los hallazgos en estos estudios reportan que las estrategias de enseñanza que se focalizan en el estudiante generan mejores aprendizajes e impactan de manera positiva en el estilo de enseñanza que adoptaría cuando se desempeñe profesionalmente (Lunenberg y Korthagen, 2003; Struyven et al., 2010). En este tipo de práctica de enseñanza la acción pedagógica se focaliza en adaptar los contenidos que se enseñan en función de las características de los estudiantes para que logren desarrollar un aprendizaje significativo de lo que se está enseñando (Depaepe et al., 2013). Se espera que los estudiantes en formación realicen este mismo proceso mediador a sus estudiantes cuando se desempeñen profesionalmente. Es por tales motivos que este tipo de conocimiento se considera un tema básico en la formación de los profesores (Borko y Putnam, 1996).

Finalmente, una práctica de enseñanza con énfasis en el desarrollo de un conocimiento académico de la enseñanza (PE-CAE) se caracteriza por tres elementos fundamentales: un tipo de conocimiento que es público, sometido a revisión crítica de la comunidad y que puede ser replicado por miembros de la comunidad en otros contextos de formación (Hutchings y Shulman, 1999; Kreber, 2002). Cabe consignar que la enseñanza en sí misma se concibe como una forma de indagación (Brew, 2006), por lo que la acción pedagógica se focaliza en compartir el conocimiento que investiga a los futuros docentes con la finalidad de difundirlo y mejorarlo. Cuando la enseñanza se concibe como una práctica investigativa en sí misma, se supera la tendencia de visualizar la enseñanza y la investigación como actividades separadas y excluyentes (Brew, 2006). Por el contrario, la investigación y la docencia se nutren mutuamente. Esto presupone una comprensión de los procesos de enseñanza que van más allá de la simple entrega de información e implica la construcción de un conocimiento acerca de la enseñanza que es mucho más complejo que demostrar una buena enseñanza (Loughran, 2006). La revisión bibliográfica efectuada refiere que este tipo de práctica de enseñanza se expresa cuando los docentes formadores vinculan docencia e investigación (Brew, 2006), o cuando los formadores investigan su propia práctica de enseñanza (Bullock, 2009; Loughran, 2007; Loughran \& Russell, 2009). En ambos casos se 
Estudios Pedagógicos, vol. XL, Número Especial 1: 161-182, 2014

POLIFONÍA DISCURSIVA Y PROCESOS DE CAMBIO EN LOS FORMADORES DE DOCENTES: ¿QUÉ VOCES MOVILIZAN SUS PRÁCTICAS DE ENSEÑANZA?

configura el doble rol asociado a los docentes formadores: su rol docente como educadores de futuros profesores y su rol de investigadores en la producción de conocimientos sobre tópicos referidos a educación, enseñanza y aprendizaje (Cochran-Smith, 2003).

\subsection{PRÁCTICA DE ENSEÑANZA DESDE UNA PERSPECTIVA DIALÓGICA: IMPLICANCIAS PARA SU ESTUDIO}

Acercarse a las prácticas de formación y enseñanza desde una perspectiva dialógica permite el análisis de la subjetividad cristalizada en los discursos, en este caso, de los formadores de profesores, en sus diversas esferas de interacción, a través de una metodología como el Análisis Dialógico del Discurso (ADD) (Ávila y Medina, 2012; Larraín y Medina, 2007; Linell, 2009; Marková ,2003; Medina, 2013). Este análisis explicita o muestra las tensiones de los agentes educativos y los efectos de ello en sus prácticas pedagógicas. El supuesto sobre el que descansa esta idea, es que la subjetividad sería una construcción polifónica compuesta por diversas voces en tensión, que estarían a la base de las decisiones y prácticas pedagógicas (Medina, En prensa).

El enfoque de ADD se basa en la idea de multivocidad de Bajtín (1982), esto es, que múltiples voces y posicionamientos ideológicos emergen en los discursos. Según Matusov (2007), la contribución central de esta idea a la educación sería enfatizar el discurso "internamente persuasivo" como meta para guiar el diseño educacional (Medina, 2013; Medina, En prensa). Para los efectos de esta investigación, las narrativas de los formadores serían consideradas como co-construcciones de su trayectoria e identidad profesional.

Esta palabra "ajena", en términos de Bajtín (1982), e internamente convincente o persuasiva, pasa a ser configuradora de la ideología personal, como mecanismo de internalización positiva de la voz del otro, relacionando y reelaborando la red de relaciones polifónicas que articulan su discurso. Por tanto, esta palabra es siempre abierta a la relación y al diálogo. Es decir, es de aceptación libre, pues es el producto de un convencimiento interno, no impuesto, donde se "adhiere" a esa otra voz. Desde esta perspectiva, el signo verbal es concebido como signo ideológico, por tanto lleva consigo una evaluación y un posicionamiento (Voloshinov, 1992). Así la palabra no es aislada, siempre remite a otras posiciones, adhiriendo a ellas o tensionándolas (Bajtín, 1989), y por ello es de carácter bivocal. De aquí que Bajtín proponga el concepto de Dialogicidad.

Por otra parte, el discurso como lugar de encuentro con el otro emerge y circula en prácticas discursivas concretas, en diversas esferas de interacción, y por tanto sujetas a la convención y formalización, plasmadas en reglas y "formas" más o menos estables de interacción social que configuran los denominados géneros discursivos. Así, el abordaje dialógico no sólo considera el discurso en su dialogización interna, sino también en estas formas estables de interacción que reclaman ciertas formas de participación y prácticas discursivas arraigadas socialmente de las que emerge, entendiendo su materialidad como el resultado de esta participación en un género y evento discursivo concreto. Esto es, no pierde de vista la emergencia social de las prácticas discursivas.

El dialogismo ofrece un modo teórico específico para concebir y analizar el discurso desde unos formatos histórico- culturales compartidos, los géneros, y permite estudiar los fenómenos de alteridad e intersubjetividad materializados en los discursos. Más allá del trabajo de Bajtín, un supuesto básico que subyace al dialogismo es que los procesos subjetivos, intersubjetivos, las configuraciones sociales y el conocimiento son 
dinámicos, contextualizados, y consideran e integran al otro (Linell, 2009; Marková, 2003). En las ciencias sociales, los diversos análisis han puesto de relieve la importancia de la intersubjetividad como un modo de tener visiones compartidas, formas de acuerdo y consenso, poniendo al diálogo como el gran mediador de estos procesos. Se ubica así a la intersubjetividad como la meta deseable de las relaciones humanas, dejando o relegando a la alteridad, que se constituye como la génesis del habla social, a un plano que debiera superarse y no asumirse como parte de la natural forma de relación y constitución de los sujetos. Por ello, un abordaje dialógico recupera el lugar natural y central de la alteridad en los procesos sociales como base de las construcciones identitarias.

En síntesis, un abordaje dialógico de las prácticas formadoras permite el acceso a la historia de interacciones sociales y hace visibles las voces internamente convincentes a las que los formadores han adherido en su trayectoria profesional, las tensiones y acuerdos con otras voces ajenas, y la manera en que estas construcciones movilizan sus prácticas pedagógicas y le permiten un posicionamiento frente a las diversas instancias y temáticas propias de su campo de participación social y, especialmente, profesional.

\section{METODOLOGÍA}

\subsection{ENFOQUE METODOLÓGICO}

El estudio reportado en este artículo forma parte de una investigación doctoral que tuvo como propósito comprender el proceso por el cual un docente se transforma en formador de futuros profesores, tomando en cuenta la dimensión personal y profesional (Montenegro, 2013). Para ello empleó un enfoque metodológico mixto utilizándose un abordaje tanto cuantitativo como cualitativo de investigación. El objetivo específico de esta fase cualitativa fue develar el discurso pedagógico que subyace a la modalidad de enseñanza que implementa el formador cuando enseña y enseña a enseñar.

\subsection{PARTICIPANTES Y PROCEDIMIENTOS}

Los participantes fueron siete docentes formadores pertenecientes a cuatro carreras de Pedagogía en Enseñanza Básica. Esta sub-muestra fue seleccionada al azar una vez que las prácticas de enseñanza propuestas en este estudio fueron identificadas en una muestra mayor de 54 docentes formadores por medio de la aplicación de dos cuestionarios: uno sobre Prácticas de Enseñanza, diseñado especialmente para esta investigación; y el cuestionario de Enfoques de Docencia Universitaria ATI-R (Montenegro, 2013). Inicialmente, 12 formadores (4 por cada enfoque de enseñanza identificado) fueron seleccionados teniendo en cuenta dos criterios: que tuviesen distintos años de experiencia profesional como formadores, y que impartieran asignaturas tanto del área de las didácticas como pedagógicas. Sin embargo, sólo 7 formadores aceptaron participar. Cabe consignar que si bien el número de participantes en esta fase investigativa fue menor a lo proyectado, el material obtenido fue suficiente para lograr un abordaje compresivo del tema investigado. De esta forma, el grupo de formadores entrevistados está conformado por 4 mujeres y 3 hombres. Cuatro formadores impartían clases en asignaturas del área de las didácticas y tres en asignaturas de formación general. En la Tabla 1 se puede apreciar la composición de la muestra: 
Tabla 1. Características de la muestra

\begin{tabular}{|cccccc|}
\hline Caso & Género & Edad & $\begin{array}{c}\text { Grado } \\
\text { Académico }\end{array}$ & $\begin{array}{c}\text { Años de experiencia } \\
\text { como formador }\end{array}$ & $\begin{array}{c}\text { Práctica de } \\
\text { Enseñanza }\end{array}$ \\
\hline 1 & Masculino & 46 & Doctorado & 14 & PE-TC \\
2 & Masculino & 48 & Licenciado & 11 & PE-TC \\
3 & Femenino & 46 & Magíster & 3 & PE-CPC \\
4 & Femenino & 36 & Magíster & 3 & PE-CPC \\
5 & Femenino & 52 & Magíster & 6 & PE-CAE \\
6 & Masculino & 30 & Magíster & 5 & PE-CAE \\
7 & Femenino & 40 & Doctorado & 4 & PE-CAE \\
\hline
\end{tabular}

Todos los participantes firmaron un consentimiento informado de acuerdo a estándares éticos nacionales, aceptando participar en este estudio. Para la recolección de los datos se aplicaron entrevistas en profundidad con preguntas semi-estructuradas respecto del tema investigado. Para el logro de ello se diseñó un conjunto de preguntas que permitieran caracterizar las prácticas de enseñanza que adoptaban; las estrategias pedagógicas que utilizaban para enseñar a enseñar; y la reflexión de diversos temas vinculados a la enseñanza. Se destaca que todas las entrevistas fueron grabadas y con posterioridad transcritas en su totalidad para la realización de los análisis posteriores.

\subsection{ANÁLISIS DE LOS DATOS}

Se efectuó un análisis dialógico del discurso (ADD) a los corpus de entrevistas desde el enfoque de la Teoría de la Enunciación (Ávila y Medina, 2012; Larraín y Medina, 2007; Medina, 2013). Esta perspectiva teórica intenta visibilizar las distintas voces o posicionamientos que emergen en el discurso y que la persona inscribe en su relato. Lo novedoso de este análisis es que posibilita visualizar que el sujeto del discurso no es un sujeto único y protagonista de la enunciación, sino más bien diverso y plural que establece relaciones con los distintos actores (entendidos como voces) que emergen en el discurso. El primer paso del procedimiento fue segmentar las entrevistas considerando su carácter genérico en pares adyacentes (entrevistador y entrevistado), para luego proceder al análisis. Larraín y Medina (2007) proponen una modalidad teórica y metodológica para aprehender la subjetividad en el discurso por medio de la distinción de cinco elementos: el sujeto de la enunciación, el sujeto del enunciado, los locutores, los enunciadores y el sujeto discursivo. En los análisis utilizados en esta investigación se emplearon sólo las siguientes categorías:

1. Enunciadores: son puntos de vista expresados en el enunciado que configuran los distintos posicionamientos y se constituyen como los centros de elaboración ideológica al interior del discurso. 
2. Sujeto del Enunciado: es el protagonista del relato o aquel núcleo ideológico desde donde se desarrolla el relato. Este núcleo manifiesta su adhesión o distancia ideológica a la postura manifestada en los enunciadores.

3. Sujeto Discursivo: se conceptualiza como al hablante en su totalidad y que sólo se puede construir a través del recorrido textual del relato.

A su vez, se emplearon seis marcas discursivas para poder identificar la subjetividad presente en el discurso analizado: uso de pronombres personales, categorías nominales, agencialidad del verbo, subjetivemas, modalidades y ventriloquismos (Álvarez, 2001; Ávila y Medina, 2012; Kerbrat-Orecchioni, 1993; Larraín y Medina, 2007). Cabe señalar que se utilizaron estas marcas discursivas por dos motivos: ya han sido utilizadas en otros análisis dialógicos del discurso (Ávila y Medina, 2012; Martínez, Tomicic y Medina, 2012; Martínez, Tomicic y Medina, En prensa); y son las más pertinentes para los propósitos de análisis de esta investigación.

Las categorías nominales son expresiones que refieren personas y sus cristalizaciones en el relato de una manera en particular, a través de pronombres, sustantivos, grupos nominales, nombres propios y marcas verbales de personas. Los subjetivemas corresponden a inscripciones discursivas que expresan juicios de valor del sujeto, dando cuenta de su posicionamiento (Kerbrat-Orecchioni, 1993). Se identificaron tres tipos de subjetivemas: adverbial, adjetivo y verbal. Las modalidades serían “operadores pragmáticos”, que podrían materializarse en una diversidad de categorías gramaticales. Éstas indican la postura del enunciador y marcan la relación extralingüística entre los que interactúan (Ávila y Medina, 2012). En este trabajo se utilizarán las categorías que Álvarez (2001) denomina "modalidades del enunciado": Deóntica (Obligación), Epistémica (Realidad Mental), Apreciativa (Valorativos) y Aléctica (Posibilidad). También se acude a la noción bajtiniana de ventriloquismo, utilizada para mostrar la emergencia en el enunciado de la recreación de la voz de otro sujeto (Larraín y Medina, 2007).

Finalmente, se destaca que para efectos de cautelar la validez y fiabilidad de este análisis se utilizó el criterio de auditoría propuesto por Flick (2000). La estrategia que se usó fue entregar el material analizado de cada uno de los casos a una experta en este tipo de análisis para que revisara los procedimientos analíticos efectuados.

\section{RESULTADOS}

El análisis dialógico del discurso efectuado da cuenta de que en los distintos casos fue posible observar que el Sujeto Discursivo se construye en base a un conjunto de Enunciadores que varían tanto en el número, el nivel de complejidad y los diálogos que establecen entre ellos. Estos núcleos ideológicos se configuran tanto en enunciados referidos a la práctica de enseñar, como también en temas relacionados con la práctica de enseñar a enseñar.

Otro elemento interesante de señalar es que se aprecian ciertos núcleos ideológicos que se configuran reiteradamente en los distintos relatos. Por ejemplo, lo fundamental que es el dominio del contenido que se debe enseñar (casos 1, 2, 3 y 4); el modelaje como un estrategia para poder enseñar a enseñar ( $\operatorname{casos} 2,3,4,5$ y 6); la vinculación entre teoría y práctica (casos 1 y 7) y la investigación como un aporte para mejorar la docencia (casos 
Estudios Pedagógicos, vol. XL, Número Especial 1: 161-182, 2014

POLIFONÍA DISCURSIVA Y PROCESOS DE CAMBIO EN LOS FORMADORES DE DOCENTES: ¿QUÉ VOCES MOVILIZAN SUS PRÁCTICAS DE ENSEÑANZA?

6 y 7). Lo relevante de este resultado es que da cuenta de que distintos posicionamientos e ideologías acerca de la práctica de enseñar situados contextualmente se materializan en una dialogización interna del discurso del formador, incorporando la palabra ajena como un posicionamiento ideológico al que adhiere el formador, a la que también adhieren otros formadores que poseen distintas trayectorias profesionales y se han desarrollado en contextos profesionales diferentes entre sí.

Respecto del análisis dialógico del discurso en formadores con distintas prácticas de enseñanza, es posible advertir diferencias interesantes de señalar. En todos los casos es posible apreciar un posicionamiento ideológico clave que permea el discurso articulando los distintos puntos de vista. En el caso de una práctica de enseñanza con énfasis en la transmisión de conocimientos (PE-TC), se marca discursivamente como un tema clave la transmisión de información desde un profesor "experto" a un estudiante "novato". Esto se puede observar en la siguiente cita del caso 2:

Yo me concentro en los contenidos. En revisar contenidos de los alumnos eh... de Historia Universal y Geografía Universal. Qué cosas un profesor de Educación Básica, no con mención, sino que un profesor de básica en el subsector de Ciencias Sociales debe saber sobre Historia.

En esta cita es posible ver cómo se articula un posicionamiento ideológico vinculado con la transmisión de contenidos representado por el Enunciador "se deben enseñar contenidos". Al respecto, es importante notar dos marcas discursivas que imprimen este posicionamiento: la modalidad deóntica DEBEN SABER en referencia al contenido disciplinar básico que el estudiante en formación debería dominar como futuro profesor de Enseñanza Básica. Y el uso del pronombre en primera persona YO para subrayar que él, como docente formador, escoge esos contenidos básicos. Es decir, la enseñanza se concentra en las acciones y decisiones que adopta el formador que enseña, más que en el estudiante que aprende. Esta tendencia de concebir la enseñanza como una práctica discursiva más bien monológica también se observa cuando se profundiza en la práctica de enseñar a enseñar. Esto se puede evidenciar en el siguiente extracto correspondiente al caso 1:

P: ¿Podrías contarme un poco cómo tú enseñas a enseñar?

R: Yo lo trabajo con los transversales. Yo habitualmente los expongo. Se los proyecto en el data... la última versión... entonces, les digo "están, existen, no se preocupen. Ahí están los verticales. Aquí están los contenidos mínimos obligatorios. Aquí están habitualmente las actividades, los recursos".

En el segmento citado es posible ver que la práctica de enseñar a enseñar se vincula con ciertos contenidos (representado por la categoría nominal OBJETIVOS TRANSVERSALES) que se enseñan a los estudiantes. Además, nuevamente se imprime en el discurso que la acción es una transmisión desde el formador hacia el estudiante. Las marcas discursivas que dan cuenta de este sentido son el uso del pronombre en primera persona YO y los verbos en voz activa EXPONGO, PROYECTO y DIGO que representan la acción de transmitir una información desde un sujeto a otro. Incluso este posicionamiento es reforzado al final del relato por medio de un ventriloquismo que recrea un diálogo entre el formador y sus estudiantes en donde se evidencia con claridad esta modalidad discursiva unidireccional centrada en la figura del formador. 
Otro elemento interesante de consignar es que el énfasis en la trasmisión de conocimientos se asocia a un tiempo necesario para lograr trasferir esos contenidos. Para ello, los formadores utilizan diversas estrategias. Por ejemplo, el caso 1 refiere que debido a la baja cantidad de horas asignadas a su cátedra, dosifica la información que entrega. Dicho posicionamiento se puede apreciar en el siguiente fragmento:

Mi curso lo debo dictar en dos horas pedagógicas a la semana...es poco tiempo. Por lo tanto, claro, reviso algunos y la revisión es habitualmente que el alumno conozca un pequeño texto.

Al interior de este relato llaman la atención dos marcas discursivas. La primera se relaciona con la percepción de escaso tiempo para poder enseñar, expresada a través de la modalidad epistémica ES asociada al subjetivema adjetivo POCO y la categoría nominal TIEMPO. La segunda marca discursiva se vincula con describir la estrategia que adopta para superar ese obstáculo: concentrar la enseñanza en la revisión de ciertos contenidos, lo que se inscribe en los verbos en voz activa REVISO y CONOZCA vinculado a la categoría nominal TEXTO presente en el relato. El caso 2 también señala esta dificultad, pero la resuelve de una forma distinta, tal como se puede apreciar en la siguiente cita:

Entonces en la cantidad de horas que tienes, que tú tienes que distribuir para todo lo que tienes. Imagínate. Desde la Prehistoria hasta la Historia Universal son dos mil años de historia. Y ver la Geografía General en seis meses. O sea, imposible. O sea, tienes que ser capaz de hacer la sintesis y marcar aquellos aspectos que son claves. Eso lo determino yo de acuerdo a mi experiencia.

Tal como se percibe en el relato, nuevamente se inscribe en el discurso la visión de escaso tiempo para poder enseñar los contenidos por medio del subjetivema adverbial IMPOSIBLE presente en el texto. Por ende, la enseñanza de los contenidos se vincula con la entrega de ciertos tópicos de la asignatura en base su experiencia como docente de aula. Dicho sentido se subjetiviza en el discurso a través del pronombre en primera persona YO (para especificar que es la persona que decide los contenidos a enseñar) y el subjetivema verbal DETERMINO asociado a la categoría nominal (mi) EXPERIENCIA inscritos al final de la cita. Estas dos últimas marcas discursivas representan la acción de seleccionar los contenidos en base a su experiencia práctica como docente de aula.

En cambio, en una práctica de enseñanza con énfasis en el desarrollo pedagógico del contenido (PC-CPC), se observa una práctica discursiva más dialógica, en donde se adapta el contenido que enseña el formador a las personas involucradas en este proceso. Por ejemplo, el caso 3 articula en su discurso un Enunciador identificado como "La enseñanza es dialogal", que postula que los procesos de enseñanza y aprendizaje se dan por medio de un diálogo con un otro. La siguiente cita ilustra lo anteriormente expuesto:

¿Cuál es la diferencia entre tú y el libro? Es que tú tienes distintas formas de explicar lo mismo y eso es lo que tú tienes que desarrollar. Sobre todo en matemáticas... las distintas formas que tienes de decir lo mismo hasta que el chico te entienda... Hasta que el chico te entienda.

En este texto se considera relevante relevar tres aspectos inscritos en el discurso. En primer lugar, se marca discursivamente que la enseñanza es un proceso interactivo entre 
Estudios Pedagógicos, vol. XL, Número Especial 1: 161-182, 2014

POLIFONÍA DISCURSIVA Y PROCESOS DE CAMBIO EN LOS FORMADORES DE DOCENTES: ¿QUÉ VOCES

MOVILIZAN SUS PRÁCTICAS DE ENSEÑANZA?

un docente y un estudiante, por medio del uso del pronombre TÚ que representa la figura del profesor y la categoría nominal CHICO que representa la figura del estudiante. En segundo lugar, la presencia de distintos roles vinculados a las personas que participan en esta interacción, lo que se inscribe por medio de las construcciones verbales EXPLICAR (asociada a la figura del docente) y ENTIENDA (asociada a la figura del estudiante). En tercer lugar, que este tipo de práctica es una actividad que distingue a docentes y formadores. Esta característica se marca por medio del pronombre personal TÚ (en referencia al estudiante en formación) y la modalidad deóntica TIENES (que desarrollar) inscritos en la cita.

Este posicionamiento de naturaleza más dialógica que incorpora al estudiante como un actor clave en este proceso también se aprecia cuando se profundiza en la práctica de enseñar a enseñar. Esta perspectiva se puede observar en el siguiente relato del caso 4:

\begin{abstract}
Porque a veces los alumnos tienden a reproducir una clase por fuera bien constructivista, pero por dentro bien tradicional. $Y$ a veces yo me pongo catete adrede para demostrarles que de manera tradicional la cosa no siempre funciona... Y ahí los estudiantes se dan cuenta que enseñar no implica sólo transmitir el contenido, sino que el otro aprenda. Y ese aprendizaje depende de las características del otro que uno como profesor debe ser capaz de identificar.
\end{abstract}

En esta cita se considera interesante relevar varias marcas discursivas. Primero, que tanto el formador como el estudiante en formación se configuran al interior del discurso a través del pronombre en primera persona YO que personifica a la formadora y la categoría nominal ESTUDIANTE que simboliza al profesor en formación. Cabe señalar que ambos son distintos actores que comparten una tarea común: la formación de otras personas. Segundo, la docente formadora tensiona que la forma de enseñar desde una perspectiva tradicional (entendida como la reproducción de información) no siempre genera resultados adecuados. Esto se puede detectar por medio de la categoría nominal MANERA TRADICIONAL vinculada a la modalidad epistémica (no) FUNCIONA y el uso del subjetivema verbal TRANSMITIR y la construcción verbal APRENDA, para marcar esta diferencia presente en el segmento. Y, tercero, se marca discursivamente que para lograr el aprendizaje en un estudiante es fundamental reconocer las principales características del educando y así poder adaptar la enseñanza. Esto se inscribe discursivamente en el pronombre indefinido UNO; la modalidad deóntica DEBE (ser) y los subjetivemas CAPAZ e IDENTIFICAR presentes al final del texto. Lo relevante de esas marcas discursivas es que connotan la práctica de enseñar como una actividad mediatizada tanto por el formador como por sus estudiantes. En otras palabras, que los procesos de enseñanza y aprendizaje están mediatizados por una práctica discursiva entre personas involucradas en este proceso.

Otro elemento significativo de consignar en el discurso de estos casos es el recuerdo de la imagen de un profesor que enseñaba dictando. El recuerdo de este tipo de práctica de enseñanza emerge discursivamente a través del Enunciador "Los profesores dictan" identificados en las dos entrevistas. El caso 3 refleja esta imagen en la siguiente cita:

Y era muy crítica con los profesores. Era pesaa. Yo les decía “ ¿Y esta clase profesora? ¿Se demoró dos horas para dictarme un libro?” Y así como qué lata.

Tal como se aprecia en el texto, el recuerdo de un profesor que enseñaba dictando se inscribe discursivamente por medio de un ventriloquismo en donde se recrea una 
interpelación de la formadora hacia su profesora en su época de estudiante en el sistema escolar. Observe cómo el relato de este recuerdo pone de manifiesto la imagen de una modalidad de enseñanza de naturaleza monológica que se concentra en la transmisión de contenidos por parte del profesor. Así, en el relato se imprime discursivamente una valoración negativa de esta forma de abordar la enseñanza a través de la expresión QUÉ LATA, con valor de subjetivema, al final del relato. Lo interesante de ello es que en ambos casos estimula a las formadoras a enseñar de manera opuesta adaptando la forma de abordar la enseñanza de acuerdo al estudiante con el que se está interactuando. Esto se puede visualizar en el siguiente ejemplo correspondiente al caso 4:

Por ejemplo eh... la metodología la veo más cercana con cosas más de la cotidianeidad. Ni siquiera de la clase. Para entender conceptos uso la realidad de cada uno de ellos.

En el relato de este ejemplo es posible identificar distintas marcas discursivas que imprimen la importancia de contextualizar y hacer pertinente la enseñanza a los estudiantes. Por ejemplo el pronombre en tercera persona ELLOS para configurar la figura del estudiante en el discurso. También el subjetivema adverbial MUCHO MÁS (cercana) y las categorías nominales COTIDIANEIDAD y REALIDAD para marcar que se utilizan distintas estrategias orientadas a adaptar el contenido dependiendo del contexto educativo y estudiante al cual se enseña. En otras palabras, nuevamente se cristaliza la noción de la enseñanza como un proceso interactivo entre profesor y estudiante en donde se deben contextualizar los contenidos que se enseñan.

Finalmente, una práctica de enseñanza con énfasis en el desarrollo de un conocimiento académico de la enseñanza (PC-CAE) también posee una naturaleza dialógica, pero asociada a un mayor grado de complejidad. Esto se sustenta debido a que no sólo incorpora al estudiante en su configuración, sino que también en aspectos contextuales que impactan en este proceso. Por ejemplo, un eje articulador a lo largo del relato del caso 5 es la importancia de desarrollar prácticas reflexivas. Esto se refleja en la siguiente cita en donde invita a un estudiante en formación a que reflexione respecto de la complejidad de enseñar. Dicho posicionamiento se articula por medio del Enunciador "Hacer clases es más que una planificación" representado por medio de un ventriloquismo que recrea la voz de la formadora interactuando con un estudiante:

Porque los alumnos, ponte tú, lo que tratan de hacer es de cumplir, de cumplir una planificación. "No me importa". Yo le digo "Si tú me entregas una planificación y yo veo que ocurre algo en el minuto en la sala y tú tienes que resolverlo. Tienes que resolverlo y parar la clase. Hay cosas más importantes que entregar esto".

Lo significativo de este posicionamiento es que enfatiza que enseñar no se debe restringir sólo a la planificación de una clase sino que también al desarrollo integral del educando. Esto se marca discursivamente en el pronombre en segunda persona TÚ (en referencia al estudiante en formación), la modalidad deóntica expresada en la forma verbal TIENES y en el verbo RESOLVERLO, operando como subjetivema, remarcado dos veces a lo largo del texto. Esto cobra relevancia si se toman en cuenta dos ideas. Por un lado, se incorporan dialógicamente elementos contextuales emergentes como otro elemento para tener en cuenta en el proceso formativo. Y por otro, la práctica de enseñar se enmarca 
Estudios Pedagógicos, vol. XL, Número Especial 1: 161-182, 2014

POLIFONÍA DISCURSIVA Y PROCESOS DE CAMBIO EN LOS FORMADORES DE DOCENTES: ¿QUÉ VOCES MOVILIZAN SUS PRÁCTICAS DE ENSEÑANZA?

como un proceso complejo que implica resolver problemas que surgen en los contextos de enseñanza, entre otras cosas.

Desde otro punto de vista, el caso 7 configura en su discurso un Enunciador identificado como "Se enseña en base a una teoría Contextualizada". Lo relevante de este Enunciador es que da cuenta que la teoría educativa que se enseña se debe contextualizar encarnándose en personas vinculadas a un contexto específico. Esto se evidencia en la siguiente cita:

Y después esos recursos tienen que estar de la mano con esa teoría pero a la vez eh... a la vez pensado para niños con... qué sé yo. Con nombres y apellidos. O sea de un centro educacional. De un contexto específico, etc.

En este texto la formadora utiliza la expresión verbal TIENEN (que estar de la mano), como una modalidad deóntica para inscribir distintos elementos claves de considerar cuando se enseña, representado por las categorías nominales NIÑOS, NOMBRES, APELLIDOS, CENTRO EDUCACIONAL y CONTEXTO inscritos en el relato. En conjunto, esas marcas discursivas dan cuenta de un mayor grado de alteridad en las redes de relaciones sociales, ya que no sólo se configura discursivamente el estudiante en formación, sino que también el contexto educacional en donde se desempeñarán profesionalmente. Por lo tanto, la práctica de enseñar debería estar orientada en esa dirección.

De manera similar, el discurso pedagógico del caso 6, cuando enseña a enseñar, posee un grado de dialogicidad que es más evidente aún, ya que trasciende el programa de formación, incorporando a la escuela como otro elemento clave para la enseñanza. En la siguiente cita se ejemplificará lo anteriormente expuesto:

Sin embargo, cuando yo superviso prácticas. Y ya uno va a verlos dos, tres veces... cuesta. Porque... y me lo he cuestionado. Que parece que a lo mejor estos cuatro, cincos años, no impactaron de tal forma en el estudiante. Porque cuando uno lo ve haciendo clases. Cuando llego sin aviso a ver una clase de un alumno en práctica y se da cuenta de que está replicando los mismos patrones o modelos de cuando él estudió. Uno dice parece que no fue tan, tan poderoso el impacto que queríamos ver en la Universidad... Y ahí viene la parte del cuestionamiento.

En este segmento se evidencia la reflexión que el caso 6 efectúa sobre su rol como docente formador. Lo interesante de esta reflexión es que cristaliza por medio de un Enunciador identificado como "Se enseña en forma tradicional” que representa una práctica de enseñanza con énfasis en la transmisión de información. Este posicionamiento se inscribe por medio de las expresiones REPLICANDO y MISMOS, con valor de subjetivemas asociados a la categoría nominal MODELOS, insertos en el relato. Cabe señalar que este núcleo ideológico tensiona al sujeto de este enunciado. En el relato es posible distinguir que el formador interpela y tensiona su propia práctica pedagógica como docente a través de la construcción verbal con valor reflexivo: ME LO HE CUESTIONADO, y la categoría nominal CUESTIONAMIENTO, presentes en el texto. También es posible apreciar que se concibe débil el impacto de la formación docente inicial entregada, lo que se marca discursivamente a través de la expresión PARECE, como modalidad apreciativa, y la construcción adjetiva TAN PODEROSO, con valor de subjetivema, asociados a la categoría nominal IMPACTO, inscritos en el relato. De este modo, los procesos formativos no se visualizan acotados a las salas de clases del programa de formación, sino que trascienden 
al contexto escolar. Esto daría cuenta de una mirada mucho más dialógica en el sentido de incorporar al estudiante y su inserción profesional como otro aspecto clave en la formación profesional de los futuros profesores.

Complementando lo anteriormente expuesto, otra temática que llama la atención en el discurso pedagógico de docentes con este tipo de prácticas de enseñanza, es que visualizan a los profesores en formación como fuente de aprendizaje para su rol como docente formador. Es decir, que aprenden a ser formadores también con sus propios estudiantes. Las siguientes citas ejemplifican esta idea:

... el hecho de estar atenta a lo que ellos necesitan me permite mejorar a mí la docencia y hacer cursos efectivamente para que salgan buenos... buenos profesores. (Caso 7)

Soy muy duro. Soy muy drástico. Muchas veces eso a los alumnos les... les choca. Les molesta que un profesor sea duro y drástico con ellos y dicen "¿por qué profesor no es un poco más flexible?” y cuando yo sé que perfectamente podría ser más flexible. Y... y tengo que cambiarlo. (Caso 6)

Tal como se aprecia en los textos citados, en los casos 6 y 7 es posible ver que los docentes formadores mejoran sus prácticas pedagógicas ya sea tomando en cuenta las inquietudes o necesidades de sus estudiantes (Caso 7), o reflexionando acerca de una retroalimentación entregada por los estudiantes orientada a la necesidad de generar un cambio en su estilo de enseñanza (Caso 6). En el caso 7, las marcas discursivas que imprimen ese posicionamiento son la construcción ESTAR ATENTA, que funciona como atributo predicativo, y en la construcción reflexiva ME PERMITE, vinculados al subjetivema verbal MEJORAR. Para el caso 6 se aprecian varias marcas discursivas vinculadas a este posicionamiento. Entre ellas se destaca la construcción verbal en primera persona SOY, con modalidad epistémica, vinculada a las construcciones adjetivas MUY DURO y MUY DRÁSTICO como atributos predicativos con valor de subjetivema (que representa la visión entregada por los estudiantes) y el verbo en modalidad deóntica TENGO, asociado a la expresión verbal CAMBIARLO, con valor de subjetivema (en referencia a la necesidad de evaluar un aspecto de su práctica de enseñanza).

En síntesis, el análisis dialógico del discurso llevado a cabo ha permitido dar una mirada más comprensiva respecto de las prácticas de enseñanza en los formadores, revelando su complejidad y los distintos elementos que es necesario tener en cuenta. En este sentido, un aspecto interesante de señalar es que más que el predominio de una práctica monológica o dialógica en los distintos enfoques de enseñanza, es posible apreciar que el grado de dialogicidad va aumentando según la forma de abordar la enseñanza que prevalece en el formador. De este modo, en una práctica de enseñanza PE-TC, en el discurso se aprecia que el dominio del contenido ocupa un lugar fundamental en el proceso formativo. En una práctica de enseñanza PE-CPC, además del contenido se incorpora discursivamente la figura del estudiante como un actor clave en este proceso. Mientras en una práctica de enseñanza PE-CAE en el relato se configuran los dos elementos anteriormente citados más la visión de la complejidad del contexto educativo en donde los estudiantes en formación se insertarán laboralmente. Algo similar se observa en el grado de complejidad cuando los formadores enseñan a enseñar. En este sentido, un aspecto revelador es que en los distintos análisis efectuados no fue posible identificar un posicionamiento ideológico que permitiese distinguir entre la práctica de enseñar y enseñar a enseñar. Si bien los formadores entrevistados informaban distintas 
estrategias pedagógicas cuando enseñaban a enseñar, no se marcó discursivamente en el relato un tipo de posicionamiento que connotara alguna diferencia entre estos procesos.

Otro resultado valioso de indicar es que, dependiendo de la forma de abordar la enseñanza, también se aprecian diferencias en la forma en que se define al estudiante y futuro profesor. Por ejemplo, en aquellas prácticas de enseñanza monológicas la figura del estudiante se releva como un actor pasivo al cual se le debe formar como profesor. En contraste, en aquellas prácticas de enseñanza más dialógicas el estudiante se concibe como una figura clave en el proceso de enseñanza con el cual también se aprende a ser formador. Como una forma de sistematizar este análisis final, en la siguiente figura se presenta un modelo comprensivo que incorpora la complejidad de la práctica de enseñanza que puede adoptar el docente formador.

Figura 1. Modelo comprensivo de las prácticas de enseñanza en docentes formadores

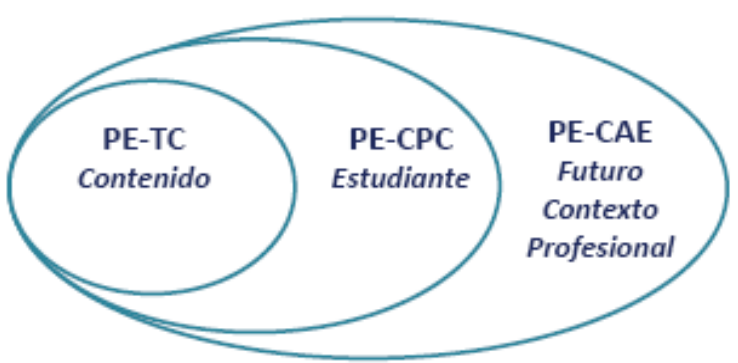

PRÁCTICAS DE ENSEÑANZA

\section{DISCUSIÓN}

El propósito de este artículo se orientó a develar el discurso pedagógico que poseen los formadores desde una perspectiva dialógica. Esto implica incorporar no sólo la voz del docente formador entendida como el resultado de un proceso de formación y desarrollo profesional, sino que también las voces internamente convincentes de personas e instituciones que han incidido en este proceso. Considerando el escaso número de investigaciones acerca de la figura del docente formador en Chile, este estudio aporta con datos empíricos importantes de tener en cuenta.

Por una parte, en los últimos años nuestro país ha desarrollado diversas políticas públicas orientadas a mejorar la formación docente inicial (Mineduc, 2005, 2009). No obstante ello, si se desea mejorar los procesos formativos que se dan al interior de esos programas, es necesario también comenzar a concebir con mayor seriedad a los actores que conducen ese proceso: los formadores de futuros profesores (Cornejo, 2007). Por otra parte, diversos estudios han demostrado la complejidad de los procesos a la base de la enseñanza cuando se enseña y enseña a enseñar (Berry, 2007; Korthagen et al., 2005; Loughran, 2006; Russell y Loughran, 2007; Zeichner, 2005). Como una forma aportar en este debate investigativo, este estudio propone un modelo conceptual para comprender tres tipos de prácticas de enseñanza que van de menor a mayor complejidad. Este modelo postula que al 
interior de los programas de formación coexisten y que a la base de ellas es posible apreciar un discurso pedagógico que sustenta la forma de comprender y llevar a cabo la enseñanza.

En este sentido, los resultados de este estudio revelan que las prácticas discursivas de los docentes formadores son distintas según la modalidad de enseñanza que prevalece y modulan su acción pedagógica cuando enseñan y enseñan a enseñar. También fue posible constatar que el sujeto discursivo se construye en base a un conjunto de enunciadores que varían tanto en el número, nivel de complejidad y diálogos que establecen entre ellos. Se identificaron ciertos núcleos ideológicos que se configuraron reiteradamente en los distintos relatos. Por ejemplo, lo fundamental que es el dominio del contenido que se debe enseñar; la vinculación entre teoría y práctica; la práctica investigativa como un aporte para la docencia; y el modelaje como una estrategia para poder enseñar a enseñar. No obstante ello, el grado de profundidad, complejidad y alcance vinculado a su configuración y posicionamiento es diferente si se asocia con la práctica de enseñanza que prevalece en el formador.

Lo interesante de este resultado es que posibilita visualizar las formas de abordar la enseñanza como una combinación de las distintas prácticas de enseñanza conceptualizadas en el modelo teórico que propone este estudio, y que los formadores poseen un discurso polifónico en el cual se integran distintas ideologías a la base de la enseñanza que dialogan, se interpelan y tensionan entre sí. En otras palabras, que la polifonía discursiva del formador da cuenta de distintas voces (entendidas como posicionamientos ideológicos) en tensión, las cuales sustentan sus decisiones y prácticas pedagógicas cuando enseña y enseña a enseñar (Medina, En prensa). Y que esta amalgama de posicionamientos se puede apreciar en el sujeto discursivo que emerge a lo largo de todo su relato como docente formador (Larraín y Medina, 2007).

Otro resultado importante de consignar es que en la totalidad de los casos se constituyó un enunciador clave que permea el discurso articulando los distintos posicionamientos que emergen en el relato. Lo relevante de este eje articulador es que a la base es posible identificar un núcleo ideológico de naturaleza más monológica o dialógica en la práctica discursiva que engloba al sujeto discursivo. En consecuencia, es posible señalar que las prácticas de enseñanza poseen distintos grados de alteridad que van aumentando según la forma de abordar la enseñanza que prevalece en el formador (Wertsch, 1999).

Por ejemplo, en una práctica de enseñanza con énfasis en la transmisión de conocimientos (PE-TC) se aprecia en el discurso que el dominio del contenido ocupa un lugar fundamental en el proceso formativo. Por ende, la voz del formador cobra protagonismo generando una práctica discursiva más bien monológica sustentada en la idea de que los estudiantes deben dominar ciertos contenidos claves para poder ejercer como profesor. A su vez, en una práctica de enseñanza con énfasis en el desarrollo de un Conocimiento Pedagógico del Contenido (PE-CPC), además del contenido que los futuros profesores deben saber, se incorpora discursivamente la figura del estudiante como un actor clave en este proceso. Por ende, se considera fundamental para el aprendizaje adaptar pedagógicamente el contenido en función de las características de los estudiantes, generándose un discurso de naturaleza más dialógica. Mientras en una práctica de enseñanza con énfasis en el desarrollo de un Conocimiento Académico de la Enseñanza (PE-CAE), en el relato se configuran los dos elementos anteriormente citados más la visión de la complejidad del contexto educativo en donde los estudiantes en formación se insertarán laboralmente.

Lo relevante de este hallazgo es que invita a visualizar los procesos formativos de manera más amplia, incorporando no sólo al estudiante en formación, sino que también 
Estudios Pedagógicos, vol. XL, Número Especial 1: 161-182, 2014

POLIFONÍA DISCURSIVA Y PROCESOS DE CAMBIO EN LOS FORMADORES DE DOCENTES: ¿QUÉ VOCES

MOVILIZAN SUS PRÁCTICAS DE ENSEÑANZA?

el sistema escolar en el cual se desempeñará en el futuro. En este sentido, la formación de los futuros profesores no debería restringirse a lo enseñado al interior de los programas de formación. Por el contario, la formación debería trascender las salas de clases universitarias, enseñando en dirección al futuro contexto profesional en donde se insertará profesionalmente el futuro profesor (Montenegro, Medina y Valdivia, 2013). Dado que al interior de los programas de formación coexisten distintos discursos pedagógicos que poseen distintos grados de complejidad y dialogicidad, un desafío para los formadores de futuros profesores es intencionar la implementación de aquellas prácticas pedagógicas más complejas que incorporan esta mirada más dialógica en los procesos formativos.

En relación a lo anteriormente expuesto, una contribución relevante de este estudio es el aporte que los conceptos de alteridad y dialogicidad pueden entregar en la comprensión de la enseñanza. Los discursos y las palabras no son neutros, ni monologales, sino co-construidos en las prácticas socio-discursivas desde donde emergen y a su vez configuran, en este caso, los procesos de formación de docentes. Es en este tejido que es posible descubrir, mediante una mirada analítica dialógica, la red de relaciones y tensiones que movilizan las prácticas pedagógicas de los formadores, y que se han ido configurando con el aporte de las diversas voces internamente convincentes y aquellas con las que se tensiona sus posturas. Resultó interesante mostrar aquellos sujetos discursivos, es decir, aquel enunciador que se posicionó predominantemente en el discurso de los formadores y su relación con los tipos de prácticas de enseñanza, revelando una relación positiva entre dialogicidad y complejidad.

En este sentido, comprender la enseñanza como una práctica con mayor o menor grado de dialogicidad se puede efectuar desde dos perspectivas: ligado al proceso de enseñanza y a las personas que participan en él. Desde la primera perspectiva se comprende la enseñanza como situada en un contexto específico que incorpora los diversos elementos que confluyen en él. Los resultados de esta investigación indican que este aumento en la complejidad va en un continuo complementando el dominio del contenido, la figura del estudiante como un actor clave en este proceso y la complejidad del contexto educativo en donde los estudiantes en formación se insertarán laboralmente. Desde la segunda perspectiva, la figura del estudiante dialógicamente se complejiza en la medida que se constituye como una figura que cumple un doble rol: es el sujeto a quien el formador enseña pero, al mismo tiempo, del cual aprende a ser formador. Uno de los hallazgos más significativos de esta investigación apunta a ese fenómeno: los formadores con prácticas de enseñanzas más complejas definen a sus estudiantes como una fuente de aprendizaje. Ellos aprenden y mejoran su rol como formadores cuando incorporan nuevos conocimientos por inquietudes o preguntas de sus estudiantes, o modifican prácticas de enseñanza en virtud de una reflexión realizada en conjunto con ellos. Por lo tanto, los programas de formación son espacios de aprendizaje no sólo de los estudiantes, sino que también de los formadores que participan en él (Montenegro, 2013).

En relación a las limitaciones de este estudio es importante mencionar que la muestra utilizada es pequeña y no representa a la totalidad de formadores que se desempeñan en ese contexto educativo. En este sentido, esta investigación siempre se enmarcó como un estudio exploratorio orientado a profundizar en el discurso pedagógico de los formadores más que prescribir lo que realizan o debieran hacer, y si bien los resultados obtenidos no pueden ser generalizados, sí contribuyen con la generación de nuevos conocimientos que permiten dar sugerencias útiles para el campo de la formación inicial en general, y el rol de los docentes formadores en particular. 
En primer lugar, se considera fundamental implementar el desarrollo de políticas y estrategias de apoyo en el aprendizaje profesional del formador. Para ello se recomienda comenzar a visualizar los programas de formación como comunidades de aprendizaje orientadas a la reflexión y producción de conocimientos de los formadores y profesores en formación (Cochran-Smith, 2003). Por ende, es primordial un cambio institucional orientado a la conformación y validación de este espacio e instalar la lógica del aprendizaje profesional entre formadores y entre el formador y sus estudiantes (Loughran, 2006).

En segundo lugar, se propone que al interior de estos espacios de aprendizaje de los formadores se potencien aspectos débiles identificados en la formación de estos profesionales. Por ejemplo, la sistematización de un lenguaje común respecto del conocimiento a la base de la práctica de enseñar que posibilite su difusión, evaluación e investigación (Loughran, 2006; Lunenberg et al., 2007). El desarrollo de trabajos colaborativos y seminarios orientados discutir la práctica de enseñar a enseñar que distingue la labor del formador (Montenegro, 2013; Murray y Male, 2005). Finalmente, la inclusión del estudiante en formación como otro miembro clave de la comunidad que también aporta en el desarrollo del conocimiento acerca de la enseñanza (Cochran-Smith, 2003).

En tercer lugar, se considera fundamental la generación de fondos que financien de manera sistemática una línea de investigación abocada al estudio de las prácticas de enseñanzas que se adoptan al interior de los programas de educación y su impacto en la formación del profesorado. Dicha política contribuiría no sólo con el desarrollo de cuerpos de conocimientos esenciales en esta área, sino que también con la función de los programas de formación como centros de producción de conocimientos acerca de la enseñanza. Dentro de los temas que se sugiere comenzar a investigar se recomiendan aquellos en donde se investigue al formador y profesor en formación de manera conjunta. También aquellos orientados al desarrollo de modelos de acompañamiento para el desarrollo de la práctica de enseñar a enseñar en los docentes formadores, y en qué medida el aumento del grado de dialogicidad permitiría mejorar los procesos formativos en los futuros profesores.

Se concluye que las principales implicancias teóricas, prácticas e investigativas de este estudio apuntan a la necesidad de preocuparnos más por la figura del formador y sus principales desafíos en la tarea de formar a futuros profesores. Los resultados de este estudio revelan que es una tarea aún más compleja de lo que uno podría suponer. Por lo tanto, si queremos contribuir con fortalecer la figura del formador, debemos comenzar por escuchar su voz, aprender de su experiencia en el campo de la formación y su posicionamiento ideológico cuando enseña y enseña a enseñar. Sólo en la medida que se articulen cuerpos de conocimientos y datos empíricos permitirán el desarrollo de políticas públicas pertinentes a las necesidades y requerimientos de este grupo de profesionales abocados a la enseñanza.

\section{REFERENCIAS BIBLIOGRÁFICAS}

Álvarez, G. (2001). Textos y discursos. Concepción: Editorial Universidad de Concepción.

Ávalos, B. (2001). El desarrollo profesional de los docentes. Proyectando desde el presente al futuro. Santiago: Unesco.

Ávila, N. y Medina, L. (2012). El Análisis Dialógico del Discurso (ADD) y la teoría de la enunciación: descubriendo la tensión dialógica en los discursos de profesores secundarios chilenos. Estudios de Psicología, vol.32, n.2, 231-247. 
Estudios Pedagógicos, vol. XL, Número Especial 1: 161-182, 2014

POLIFONÍA DISCURSIVA Y PROCESOS DE CAMBIO EN LOS FORMADORES DE DOCENTES: ¿QUÉ VOCES

MOVILIZAN SUS PRÁCTICAS DE ENSEÑANZA?

Bajtín, M. (1982). Estética de la creación verbal. México: Siglo Veintiuno.

Bajtín, M. (1989). Teoría y estética de la novela. Madrid: Altea, Taurus, Alfaguara.

Berry, A. (2007). Tensions in teaching about teaching: Understanding practice as a teacher educator. Dordrecht: Springer.

Berry, A. (2009). Professional self-understanding as expertise in teaching about teaching. Teachers and Teaching: Theory and Practice, vol.15, n.2, 305-318.

Borko, H. (2004). Professional development and teacher learning: mapping the terrain. Educational Researcher, vol.33, n.8, 3-15.

Borko, H., \& Putnam, R. (1996). Learning to teach. In D. Berliner y R. Calfee (Eds.), Handbook of Educational Psychology (pp. 673-708). New York: Macmillan.

Brew, A. (2006). Research and Teaching: Beyond the Divide. New York: Palgrave Macmillan.

Bullock, S.M. (2009). Learning to think like a teacher educator: making the substantive and syntactic structures of teaching explicit through self-study. Teachers and Teaching: Theory and Practice, vol.15, n.2, 291-304.

Cisternas, T. (2011). La investigación sobre formación docente en Chile. Territorios explorados e inexplorados. Calidad en la Educación, n.35, 131-164.

Cochran-Smith, M. (2003). Learning and unlearning: the education of teacher educators. Teaching and Teacher Education, vol.19, n.1, 5-28.

Cochran-Smith, M., \& Lytle, S. (1999). Relationships of Knowledge and Practice: Teacher Learning in Communities. Review of Research in Education, vol.24, n.1, 249-305.

Cornejo, J. (2005). La formación de formadores de profesores: una deuda ineludible, un desafío pendiente. Foro Educacional, n.8, 15-45.

Cornejo, J. (2007). La formación de los formadores de profesores: ¿Para cuándo en Chile? Revista Pensamiento Educativo, vol.41, n.2, 37-55.

Dall'Alba, G., \& Sandberg, J. (2006). Unveiling Professional Development: A Critical Review of Stage Models. Review of Educational Research, vol.76, n.3, 383-412.

Darling-Hammond, L. (2006). Powerful Teacher Education: Lessons from Exemplary Programs. San Francisco: Jossey-Bass.

Darling-Hammond, L., \& Bransford, J. (2005). Preparing Teachers for a Changing World. What teachers should learn and be able to do. San Francisco: Jossey-Bass.

Depaepe, F., Verschaffel, L., \& Kelchtermans, G. (2013). Pedagogical content knowledge: A systematic review of the way in which the concept has pervaded mathematics educational research. Teaching and Teacher Education, vol.34, n.1, 12-35.

Dinkelman, T., Margolis, J., \& Sikkenga, K. (2006). From teacher to teacher educator: experiences, expectations, and expatriation. Studying Teacher Education: A journal of self-study of teacher education practices, vol.2, n.1, 5-23.

Flick, U. (2000). Introducción a la investigación cualitativa. Madrid: Ed. Morata SL.

Hutchings, P., \& Shulman, L. (1999). The scholarship of teaching: New elaborations, new developments. Change, vol.31, n.5, 11-15.

Kane, R. (2007). From naive practitioner to teacher educator and researcher: constructing a personal pedagogy of teacher education. In T. Russell, \& J. Loughran (Eds.), Enacting a Pedagogy of Teacher Education. Values, relationships and practices (pp. 60-76). New York: Routledge.

Kerbrat-Orecchioni, C. (1993). La enunciación de la subjetividad en el lenguaje. Buenos Aires: Edicial.

Korthagen, F., Loughran, J., \& Lunenberg, M. (2005). Teaching teachers-studies into the expertise of teacher educators: an introduction to this theme issue. Teaching and Teacher Education, vol.21, n.2, 107-115.

Korthagen, F., Loughran, J., \& Russell, T. (2006). Developing fundamental principles for teacher education programs and practices. Teaching and Teacher Education, vol.22, n.8, 1020-1041.

Kreber, C. (2002). Teaching Excellence, Teaching Expertise, and the Scholarship of Teaching. 
Innovative Higher Education, vol.27, n.1, 5-23.

Larraín, A. y Medina, L. (2007). Análisis de la enunciación: distinciones operativas para un análisis dialógico del discurso. Estudios de Psicología, vol.23, n.3, 283-301.

Linell, P. (2009). Rethinking language, mind, and world dialogically: interactional and contextual theories of human sense-making. Charlotte, NC: Information Age Pub.

Loughran, J. (2006). Developing a Pedagogy of Teacher Education. Understanding teaching and learning about teaching. New York: Routledge.

Loughran, J. (2007). Researching Teacher Education Practices: Responding to the Challenges, Demands, and Expectations of Self-Study. Journal of Teacher Education, vol.58, n.1, 12-20.

Loughran, J., \& Berry, A. (2005). Modelling by teacher educators. Teaching and Teacher Education, vol.21, n.2, 193-203.

Loughran, J., \& Russell, T. (2009). Teaching as a discipline. Teachers and Teaching: Theory and Practice, vol.15, n.2, 183-187.

Lunenberg, M., \& Korthagen, F. (2003). Teacher educators and student-directed learning. Teaching and Teacher Education, vol.19, n.1, 29-44.

Lunenberg, M., Korthagen, F., \& Swennen, A. (2007). The teacher educator as a role model. Teaching and Teacher Education, vol.23, n.5, 586-601.

Marková, I. (2003). Dialogicality in the Prague School of Linguistics: a theoretical retrospect. In I. Josephs, \& J. Valsiner (Eds.), Dialogicality in development, Advances in Child Development within Culturally Structured Environments (pp. 3-33). Westport: Praeger.

Martínez, C., Tomicic, A., \& Medina, L. (2012). Dialogic discourse analysis of psychotherapeutic dialogue: microanalysis of relevant psychotherapy episodes. International Journal for Dialogical Science, vol.6, n.1, 99-121.

Martínez, C., Tomicic, A., \& Medina, L. (En prensa). Psychotherapy as a Discursive Genre: A Dialogic Approach. Culture \& Psychology.

Martinez, K. (2008). Academic induction for teacher educators. Asia-Pacific Journal of Teacher Education, vol.36, n.1, 35-51.

Matusov, E. (2007). Application of Bakhtin scholarship on discourse and education: A critical review essay. Educational Theory, vol.57, n.2, 215-237.

Medina, J.L. (2006). La profesión docente y la construcción del conocimiento profesional. Buenos Aires: Magisterio del Río de la Plata.

Medina, L. (2013). El Análisis Dialógico del Discurso: analizar el discurso sin olvidar el discurso. En Canales, M. (Coord.). Escucha de la escucha. Análisis e interpretación en la investigación cualitativa (pp. 295-317). Santiago: LOM Ediciones y Doctorado en Cs. Sociales, Universidad de Chile.

Medina, L. (en prensa). ¿Cómo acceder a las concepciones y teorías que subyacen a las prácticas docentes? Una reflexión teórico-metodológica desde el Análisis dialógico de los discursos. Cultura \& Educación.

Mineduc. (2005). Informe Comisión sobre Formación Inicial Docente. Santiago de Chile: Autor.

Mineduc. (2009). Programa INICIA. Última visita 30 de Mayo de 2009. Recuperado desde http://www.programainicia.cl

Montenegro, H. (2013). La práctica de enseñar en los Programas de Formación Docente Inicial: ¿Cómo el profesor se convierte en formador de futuros profesores? (Tesis Doctoral no publicada). Pontificia Universidad Católica de Chile.

Montenegro, H. y Fuentealba, R. (2012, Agosto). La práctica de enseñar a enseñar en la formación de futuros profesores: propuesta de un modelo para su estudio. Paper presentado en el Segundo Congreso Interdisciplinario de Investigación en Educación, Santiago, Chile.

Montenegro, H., Medina, L., \& Valdivia, A. (2013, Abril). Pedagogical discourse in teacher educators: senses and meanings. Paper presentado en el Annual Meeting of the American Educational Research Association [AERA]. Philadelphia. 
Murray, J., \& Male, T. (2005). Becoming a teacher educator: evidence from the field. Teaching and Teacher Education, vol.21, n.2, 125-142.

OECD. (2004). Revisión de Políticas Nacionales de Educación: Chile. Santiago: OCDE y Ministerio de Educación de Chile.

Robinson, M., \& McMillan, W. (2006). Who teaches the teachers? Identity, discourse and policy in teacher education. Teaching and Teacher Education, vol.22, n.3, 327-326.

Russell, T., \& Loughran, J. (2007). Enacting a Pedagogy of Teacher Education. Values, relationships and practices. New York: Routledge.

Savvidou, C. (2010). Storytelling as dialogue: how teachers construct professional knowledge. Teachers and Teaching: Theory and Practice, vol.16, n.6, 649-664.

Shulman, L. (1986). Those who understand: Knowledge growth in teaching. Educational Researcher, vol.15, n.2, 4-14.

Shulman, L. (1987). Knowledge and teaching. Foundations of the new reform. Harvard Education Review, vol.57, n.1, 1-22.

Shulman, L. (2004). Research on Teaching: A Historical and Personal Perspective. En L. Shulman, \& S. Wilson (Eds.), The Wisdom of Practice. Essays on Teaching, Learning and Learning to Teach (pp. 363-381). USA: Jossey-Bass.

Struyven, K., Dochy, F., \& Janssens, S. (2010). Teach as you preach: the effects of studentcentred versus lecture-based teaching on student teachers' approaches to teaching. European Journal of Teacher Education, vol.33, n.1, 43-64.

Swennen, A., \& Van der Klink, M. (2009). Becoming a Teacher Educator. Theory and Practices for Teacher Educators. Dordrecht: Springer.

Voloshinov, V. (1992). El marxismo y la filosofía del lenguaje. Madrid: Alianza.

Wertsch, J. (1999). La mente en acción. Buenos Aires: AIQUE.

Zeichner, K. (2005). Becoming a teacher educator: a personal perspective. Teaching and Teacher Education, vol.21, n.2, 117-124. 